\title{
Restore from Restored: Video Restoration with Pseudo Clean Video
}

\author{
Seunghwan $\mathrm{Lee}^{1}$, Donghyeon $\mathrm{Cho}^{2}$, Jiwon $\mathrm{Kim}^{3}$, Tae Hyun Kim ${ }^{1}$ \\ seunghwanlee@hanyang.ac.kr, cdh12242@gmail.com, ai.kim@sk.com, taehyunkim@hanyang.ac.kr \\ ${ }^{1}$ Dept. of Computer Science, Hanyang University, Seoul, Korea \\ ${ }^{2}$ Dept. of Electronic Engineering, Chungnam National University, Daejeon, Korea \\ ${ }^{3}$ SKT Vision AI Labs/T-Brain X, Seoul, Korea
}

\begin{abstract}
In this study, we propose a self-supervised video denoising method called "restore-from-restored." This method fine-tunes a pre-trained network by using a pseudo clean video during the test phase. The pseudo clean video is obtained by applying a noisy video to the baseline network. By adopting a fully convolutional neural network (FCN) as the baseline, we can improve video denoising performance without accurate optical flow estimation and registration steps, in contrast to many conventional video restoration methods, due to the translation equivariant property of the $F C N$. Specifically, the proposed method can take advantage of plentiful similar patches existing across multiple consecutive frames (i.e., patch-recurrence); these patches can boost the performance of the baseline network by a large margin. We analyze the restoration performance of the fine-tuned video denoising networks with the proposed selfsupervision-based learning algorithm, and demonstrate that the FCN can utilize recurring patches without requiring accurate registration among adjacent frames. In our experiments, we apply the proposed method to state-of-theart denoisers and show that our fine-tuned networks achieve a considerable improvement in denoising performance ${ }^{1}$.
\end{abstract}

\section{Introduction}

Video restoration, which aims to recover the high-quality video frames from the low-quality video, is one of the oldest research fields in video processing. Video denoising, which removes noise in the video frames, has been investigated considerably. However, estimating a clean image from a corrupted frame is a well-known inverse problem. To solve such an ill-posed problem, various types of approaches, including prior model, likelihood model, optimization, and deep learning techniques, have been introduced.

\footnotetext{
${ }^{1}$ Code is available at https://github.com/shlee0/RFRvideo-denoising
}

A common natural image property used for image restoration is patch-recurrence in which similar patches exist within a single image. Particularly, patch-recurrence has been considerably studied in single-image super-resolution (SR) methods $[9,11,12]$. Although these patches can be deformed by camera and/or object motion in a video, patchrecurrence over neighboring video frames is much richer than that of a single image and can further improve the quality of the restored frames $[19,18]$. Moreover, rich patchrecurrence information can greatly help in the fine-tuning of video restoration networks during the test stage, without using ground-truth clean images.

Lehtinen et al. [16] proposed single-image denoising method (noise-to-noise), which allows the training of the restoration network without ground-truth clean images. Ehret et al. [7] proposed a frame-to-frame training technique, which extends the noise-to-noise training algorithm for video restoration; the frame-to-frame training algorithm can also perform fine-tuning without using the ground-truth clean video by aligning noisy patches among consecutive frames using optical flow. However, estimating accurate optical flow under large displacements, occlusion, and severe degradation (e.g., noise and blur) is a challenging task. Thus, in this work, we propose a new training algorithm called "restore-from-restored," which allows fine-tuning of pre-trained networks without using the ground-truth clean video and accurate optical flow for registration.

Our proposed method updates the parameters of pretrained networks using pseudo clean images, which are outputs of the pre-trained baseline networks from noisy input frames. Our algorithm is simple yet effective for removing noise in video frames and works particularly well with the existence of numerous recurring patches. That is, we generate pairs of training images, which are composed of the pseudo clean video and its noisy versions, to fine-tune the network. In practice, pixel locations of the same patches in different video frames vary due to motions, but with the aid of the translation equivariant property of a fully convolutional network (FCN), our algorithm can update the network 
parameters without using optical flow to align the translated patches only if they are fully convolutional. We demonstrate the superiority of the proposed algorithm by applying it to state-of-the-art video denoising networks and providing improved denoising results. The contributions of this study are summarized as follows:

- We propose a novel self-supervised training algorithm to fine-tune fully pre-trained networks without using the clean ground-truth video.

- We explain why and how the proposed training scheme works with the patch-recurrence property.

- The proposed method can be easily integrated with state-of-the-art denoising networks and yields state-ofthe-art denoising results on the benchmark datasets including not only synthetic but also real noise.

\section{Related Works}

In this section, we provide a brief overview of recent works that are related to the proposed restoration algorithm, in terms of training with and without using ground-truth clean data.

Training with ground-truth clean data. When a set of high-quality images is available, we can generate synthetic degraded images, and train deep neural networks with these images and restore them to their original high-quality state.

In the case of image denoising, Xie et al. [26] applied deep neural networks to model the mapping of clean images from noisy input images. They generated pairs of noisy and clean images to train the neural networks. Since then, numerous studies on the image denoising task have been conducted using deep CNN with train pairs of clean and synthetically noisy images [28, 29, 30, 32, 15, 17, 31, 10, 1]. Several studies have adopted residual learning schemes to allow deep neural networks and extend the receptive field [28, 32]. Moreover, to incorporate long-range dependencies among pixels, several studies utilized non-local networks [31, 17]. Recent efforts have attempted to deal with unknown noise in real photographs (blind restoration). Guo et al. [10] proposed a two-stage method that consists of noise estimation and non-blind denoising steps. Gao and Grauman [8] proposed an on-demand learning method to handle various corruption levels for each restoration task including denoising, inpainting, and deblurring. These research trends have also been applied to video restoration problems. For instance, Davy et al. [6] not only incorporated non-local information with a non-local patch search module but also adopted a residual learning scheme for video denoising. FastDVDnet [25] proposed a cascaded two-step architecture with a modified multi-scale U-Net. FastDVDnet exhibits fast runtimes by avoiding costly explicit motion compensation and handling motion implicitly due to the attributes of its dedicated architecture. Yue et al. [27] proposed RViDeNet to restore real noisy video frames in raw image spaces and achieved state-of-the-art denoising performance. RViDeNet adopts deformable convolution [5] to align consecutive frames and utilizes spatiotemporal fusion stages to reconstruct the result. Moreover, Yue et al. provided a new video denoising dataset, namely, Captured Raw Video Dataset (CRVD).

Regardless of the techniques used in previous works (e.g., residual learning, non-local network, and model-blind approaches), these studies require the generation of synthetic images for network training. Thus, these supervised approaches hardly deal with datasets where clean images can be rarely obtained (e.g., medical imaging system).

Training without ground-truth clean data. Several attempts have been made recently to learn restoration networks without using ground-truth clean data. Lehtinen et al. [16] trained a network with pairs of noisy patches under the assumption that the average of many differently corrupted pixels is close to clean data. Then, Krull et al. [14] and Baston and Royer [2] introduced self-supervised single image denoising methods without relying on clean data. Ehret et al. [7] introduced a frame-to-frame training method to learn video restoration networks without clean images by extending the strategy proposed in [16] to videos. For removing noise in a certain patch, their method searches corresponding patches among adjacent frames by using optical flow and then warps the patches to create pairs of aligned noisy patches for network training. Frame-to-frame training enables the exploitation of patch-recurrence property within input video frames and fine-tune the pre-trained networks using test inputs. This approach can boost the performance of the existing pre-trained networks because networks can be further optimized without the ground-truth targets at the test stage.

However, one disadvantage of this method [7] is that accurate optical flow, which is difficult to estimate under large displacements, occlusions and serious damages, is required to fine-tune network. In this study, we overcome the limitations of [7] by using a new training scheme called "restorefrom-restored." Technically, pseudo clean images are generated from a pre-trained video restoration network and then used as train targets for fine-tuning during the test phase. It produces a synergy effect with the patch-recurrence property that appears repeatedly over consecutive video frames. In the following sections, we provide detailed analysis on the proposed method and show the proposed method can boost the performance of the fully pre-trained denoising networks with the help of the patch-recurrence property.

To the best of our knowledge, the proposed method is the first neural approach to boost the performance of pre-trained convolutional video restoration networks without using accurate registration or non-local operation while using recur- 
ring patches in the test-phase.

\section{Self-Supervised Video Restoration}

Patch-recurrence within the same scale is rich in natural images [24, 22] and becomes more redundant when multiple neighboring video frames are available [23]. To utilize this space-time recurring information among given video frames, conventional restoration methods require accurate correspondences between adjacent frames and thus need to compute the optical flow to align the neighboring frames to the reference frames $[3,21,13]$.

In this work, we present a novel yet simple training algorithm (test-time fine-tuning) that can be applied to video restoration networks. Our fine-tuning algorithm is based on self-supervision and does not require ground-truth clean images. Moreover, the proposed algorithm allows restoration networks to exploit patch-recurrence without accurate optical flow estimation and registration steps while improving performance by a large margin. Many convolutional video restoration networks, including state-of-the-art methods, can be easily fine-tuned using our self-supervised training algorithm without changing their original network architecture if they are fully convolutional.

\subsection{Restore-from-restored}

In this section, we explain how we can fine-tune and improve the performance of the pre-trained video restoration networks without using the clean video frames during the test stage.

In general, conventional video restoration networks are trained with labeled ground-truth clean images; these networks learn a function $f_{\theta}$, which maps a corrupted input frame $\mathbf{Y}$ to a clean target frame $\mathbf{X}$, where $\theta$ denotes the function parameters. Specifically, the network parameter $\theta$ is trained by minimizing the loss function $L$ between the network outcome and the training target as

$$
\operatorname{Loss}(\theta)=L\left(f_{\theta}(\mathbf{Y}), \mathbf{X}\right) .
$$

For the loss function $L$, common choices are L1 and L2 losses in many denoising approaches [2, 16]. Although image restoration networks trained by minimizing the distance between the network output and the training target can produce highly satisfactory results, these networks can be further upgraded by utilizing redundant spatio-temporal information (e.g., patch-recurrence) over neighboring video frames [13, 6]. However, optical flow estimation networks or non-local operation modules to exploit the recurring patches among different frames are expensive and require additional resources to extract the temporal information $[3,6,21,17,31]$.

Therefore, we develop a simple and effective finetuning algorithm that can exploit patch-recurrence in spacetime without explicitly searching similar patches/features through optical flow estimation or non-local operation. To achieve this goal, we assume that we have a fully pretrained network $f_{\theta_{0}}$ and use initially denoised video frames $\left\{\tilde{\mathbf{X}}_{1}, \ldots, \tilde{\mathbf{X}}_{T}\right\}$ as train targets for the fine-tuning, where $\tilde{\mathbf{X}}_{t}=f_{\theta_{0}}\left(\mathbf{Y}_{t}\right)$ and $t$ denotes the frame index. Although these denoised images are not clean ground-truth images and may include some artifacts, they can be used as pseudo clean targets to fine-tune the network parameter in our study. Using the pseudo clean images, we can synthesize pseudo noisy images by adding random noise $\mathbf{N}$ to the pseudo clean images, and the pairs of pseudo clean and pseudo noisy images can be used to fine-tune the denoising networks by minimizing the loss as follows:

$$
\operatorname{Loss}(\theta)=\sum_{t=1}^{T} L\left(f_{\theta}\left(\tilde{\mathbf{X}}_{t}+\mathbf{N}\right), \tilde{\mathbf{X}}_{t}\right),
$$

Notably, the ground-truth frames and motion flows are not used during our fine-tuning process. Nevertheless, under an assumption that the distributions of the original noisy input and the corresponding pseudo noisy images are similar, we can update the network parameter $\theta$ by minimizing the proposed loss with the initially denoised frames and their synthetically corrupted counterparts if the networks are fully convolutional. Assume that we have self-similar noisy patches $\mathbf{y}_{a}$ and $\mathbf{y}_{b}$ where $\mathbf{y}_{b}$ is a translated version of $\mathbf{y}_{a}$ by a translational motion $A$ (i.e., $A\left(\mathbf{y}_{a}\right)=\mathbf{y}_{b}$ ). Then, we can generate pseudo clean patches $\tilde{\mathbf{x}}_{a}$ and $\tilde{\mathbf{x}}_{b}$ with a pretrained network, and we see that $A\left(\tilde{\mathbf{x}}_{a}\right)=\tilde{\mathbf{x}}_{b}$ when the network is an FCN, and it yields,

$$
\begin{aligned}
L\left(f_{\theta}\left(\tilde{\mathbf{x}}_{a}+\mathbf{N}\right), \tilde{\mathbf{x}}_{a}\right) & \approx L\left(f_{\theta}\left(A\left(\tilde{\mathbf{x}}_{a}+\mathbf{N}\right)\right), A\left(\tilde{\mathbf{x}}_{a}\right)\right) \\
& \approx L\left(f_{\theta}\left(\tilde{\mathbf{x}}_{b}+\mathbf{N}\right), \tilde{\mathbf{x}}_{b}\right) .
\end{aligned}
$$

Therefore, overall loss in (2) does not depend on locations of self-similar patches over multiple frames during the back-propagation with FCNs, and our fine-tuned network predicts the averaged version of denoised self-similar patches with L2 loss when one of the self-similar noisy patches is given as input. We call this process "restorefrom-restored" training, since the proposed method is relying on the initially restored (denoised) frames. We repeat our training algorithm several times until convergence and achieve considerable improvement over the fully pretrained initial network.

\subsection{Frame-to-frame vs. Restore-from-restored}

The notion of our "restore-from-restored" algorithm is based on recent noise-to-noise training mechanism by Lehtinen et al. [16]. Noise-to-noise demonstrated that image restoration networks can be trained without using ground-truth clean data for certain types of noise (e.g., zeromean noise, such as Gaussian noise and Bernoulli noise) and can be extended into the frame-to-frame approach by Ehret et al. [7] to process a video. 
Exploiting space-time patch-recurrence. The frame-toframe training algorithm [7] allows the network to train with self-supervision during the test-stage. Specifically, the network is fine-tuned with two aligned noisy frames by minimizing the loss as

$$
\operatorname{Loss}(\theta)=\sum_{t=1}^{T} L\left(f_{\theta}\left(\mathbf{Y}_{t}\right), \mathbf{Y}_{t-1}^{w}\right),
$$

where $\mathbf{Y}_{t-1}^{w}$ denotes the warped version of the noisy frame $\mathbf{Y}_{t-1}$ and is aligned to the reference frame $\mathbf{Y}_{t}$. Thus, the calculation of optical flow for registration is necessary in (4). However, the accurate estimation of optical flow is difficult to achieve in some conditions, such as severe degradation, large displacement between frames.

By contrast, our proposed loss in (2) does not need warping and alignment. If the denoising network $f_{\theta}$ is an FCN, then our method can maintain the performance without accurate registration due to the translation equivariant nature of FCN [4]. That is, our "restore-from-restored" approach is not disturbed by the existence of large translational motions compared with optical flow-based methods.

Noise reduction. Assume that a set of perfectly aligned images $\left\{\mathbf{Y}_{1}, \ldots, \mathbf{Y}_{T}\right\}$ (e.g., burst mode images from a camera on a tripod) is given, and these images are corrupted by zero-mean Gaussian random noise whose standard deviation is $\sigma$. Using the frame-to-frame training algorithm [7], the denoised frames become an averaged version of noisy inputs $\left(=\frac{1}{T} \sum_{t=1}^{T} \mathbf{Y}_{t}\right)$ with the fine-tuned parameter, and the noise variance of the denoised frame is reduced to $\frac{1}{T} \sigma^{2}$ (refer to the Appendix in [16] for details).

By contrast, the latent frame predicted from a fine-tuned parameter by minimizing the L2 version of the proposed loss in (2) is $\frac{1}{T} \sum_{t=1}^{T} \tilde{\mathbf{X}}_{t}$. Thus, the noise variance of our denoising result becomes $\frac{1}{T} \sigma_{\theta_{0}}{ }^{2}$, where $\sigma_{\theta_{0}}$ denotes the mean standard deviation of the remaining noise in $\tilde{\mathbf{X}}_{t}$. In general, as $f_{\theta_{0}}$ is a fully pre-trained network, the noise level of the residual noise $\sigma_{\theta_{0}}$ is much lower than the original noise level $\sigma$ (i.e., $\sigma_{\theta_{0}}<<\sigma$ ). Therefore, the noise variance of the latent frame from our "restore-from-restored" algorithm is much lower than that from the frame-to-frame training. Notably, our algorithm can achieve better results when there is a rich patch-recurrence, and our algorithm can show space-time varying denoising performance. For example, noisy regions where structures are highly repeating can be restored much better than regions with slightly repeating or unique patterns.

\section{Proposed Method}

By minimizing the proposed loss function in (2), we can restore clean images from degraded ones in offline and online manners, similar to [7]. In the offline video denoising
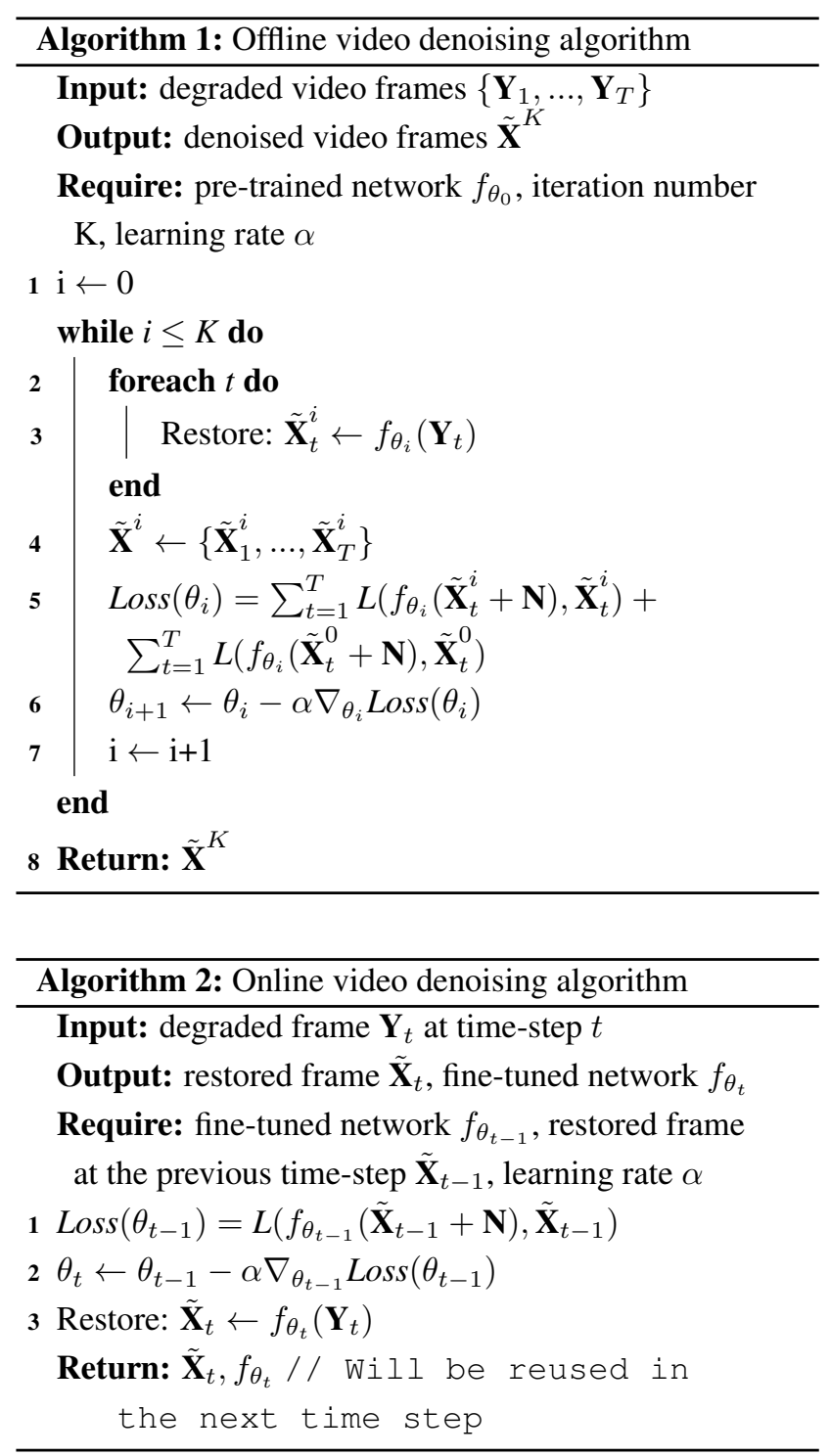

mode, we can take advantage of all the given frames for fine-tuning the network parameter. In the online denoising mode, fine-tuning and denoising are conducted successively in a sequential fashion (frame-by-frame).

Offline denoising. The offline video denoising algorithm is elaborated in Algorithm 1. For offline fine-tuning at each train step $i$, we remove the noise in the input noisy images $\left\{\mathbf{Y}_{1}, \ldots, \mathbf{Y}_{T}\right\}$ using denoising network $f_{\theta_{i}}$ and obtain denoised images $\left\{\tilde{\mathbf{X}}_{1}^{i}, \ldots, \tilde{\mathbf{X}}_{T}^{i}\right\}$. Next, we use pairs of training images $\left\{\left(\tilde{\mathbf{X}}_{1}^{i}+\mathbf{N}, \tilde{\mathbf{X}}_{1}^{i}\right), \ldots,\left(\tilde{\mathbf{X}}_{T}^{i}+\mathbf{N}, \tilde{\mathbf{X}}_{T}^{i}\right)\right\}$ to fine-tune the network parameter by minimizing the loss function in (2). To avoid over-fitting and generating over-smoothed results, we use the initially denoised frames $\left\{\tilde{\mathbf{X}}_{1}^{0}, \ldots, \tilde{\mathbf{X}}_{T}^{0}\right\}$ as additional train targets during the fine-tuning stages. In our sup- 
plementary material, we demonstrate the effect of the second loss term in Algorithm 1 by comparing results with and without using the initial frames during offline fine-tuning when $K$ is large.

Online denoising. We can also adapt the network parameter in a sequential manner. Our online video denoising algorithm is given in Algorithm 2. In contrast to the offline denoising mode, our online denoising algorithm uses only a previous frame for the update at each time step. To do so, we slightly modify the proposed loss function in (2) for the time step $t$, to take a single pair of images $\left(\tilde{\mathbf{X}}_{t-1}+\mathbf{N}, \tilde{\mathbf{X}}_{t-1}\right)$ which is previous pseudo clean and noisy frames. In Algorithm 2 , temporally varying parameter $\theta_{t}$ denotes the finetuned network parameter at time $t$; thus, each frame is denoised with a different network parameter.

\section{Experiments}

In our experiments, we apply our offline and online denoising algorithms to conventional denoising networks, including state-of-the-art video denoising methods, and evaluate the denoising performance quantitatively and qualitatively. Please refer to our supplementary material for additional experimental results, including video clips.

\subsection{Implementation Details}

We use officially available fully pre-trained network parameters for our baseline denoisers. During the fine-tuning period, we minimize the L2 loss for the proposed offline and online denoising algorithms. We also use Adam for the updates, with a learning rate of $1 \mathrm{e}-5$. We measure the performance in terms of PSNR and SSIM on RGB color channels for the objective evaluation.

First, for Gaussian noise removal, we use VNLnet [6] and FastDVDnet [25]. VNLnet is a state-of-the art video denoising method and shows the best denoising performance. Although VNLnet is integrated with the non-local operation module, we show that our algorithm can be easily applied to networks with the non-local module and can further improve the performance of the baseline networks. For the evaluation, we use 7 video clips consisting of 100 sequences each on the Derf database ${ }^{2}$ and additional 7 video clips in the DAVIS video segmentation challenge dataset [20]. We use down-scaled video frames $(960 \times 540)$, and generate noisy input videos by adding Gaussian random noise with different noise levels $(\sigma=15,25,40)$.

Next, for real noise removal, we use RViDeNet [27] and evaluate on the CRVD dataset [27]. We improve the baseline performance with the proposed fine-tuning algorithm in the sRGB space. To handle real noise, we synthesize random noise $\mathbf{N}$ with the noise model used in the pre-training

\footnotetext{
${ }^{2}$ https://media.xiph.org/video/derf/
}

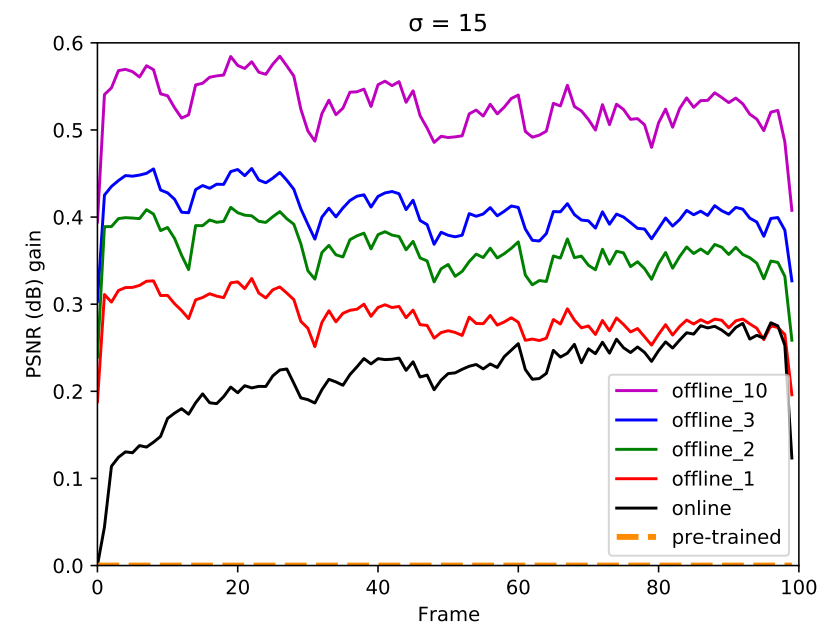

Figure 1: Performance gains from our online and offline denoising algorithms. Baseline network is FastDVDnet [25]. Differences of PSNR values before and after fine-tuning are measured on Derf datasets on $\sigma=15$. Number $i$ in "offline $\_l$ " denotes the number of steps (i.e., $K$ ). Note that the rapid rise and drop of performance at the very first and last time step are due to a usability of adjacent frames.

process of RViDeNet. Refer to our supplementary material for detailed settings.

\subsection{Denoising Performance}

Quantitative results. First, we fine-tune FastDVDnet [25] on the Derf dataset with the proposed offline and online denoising algorithms to evaluate the Gaussian denoising performance; the performance gains are depicted in Fig. 1. We achieve consistently improved denoising performance over the baseline as the number of iterations (i.e., $K$ ) increases with the offline denoising algorithm. Meanwhile, we obtain improved results as the frame number increases with our online denoising algorithm because the proposed online method enables sequential parameter update. In Tab. 1 and Tab. 2, we provide the PSNR and SSIM values of the denoising results from our algorithms on the Derf and DAVIS testsets. These results show that the online and offline denoising algorithms can produce steadily better results than the baseline models, and the offline denoising algorithm with 10 updates (i.e., $K=10$ ) achieves the best performance. In Tab. 3, we add quantitative denoising results on the real noise dataset, CRVD [27]. As CRVD includes only short sequences $(T=7)$, we only evaluate the performance of the offline restoration algorithm; the results show that the proposed learning algorithm can effectively remove real noise remarkably and enhance the denoising results.

Visual results. In Fig. 2, we provide qualitative compari- 

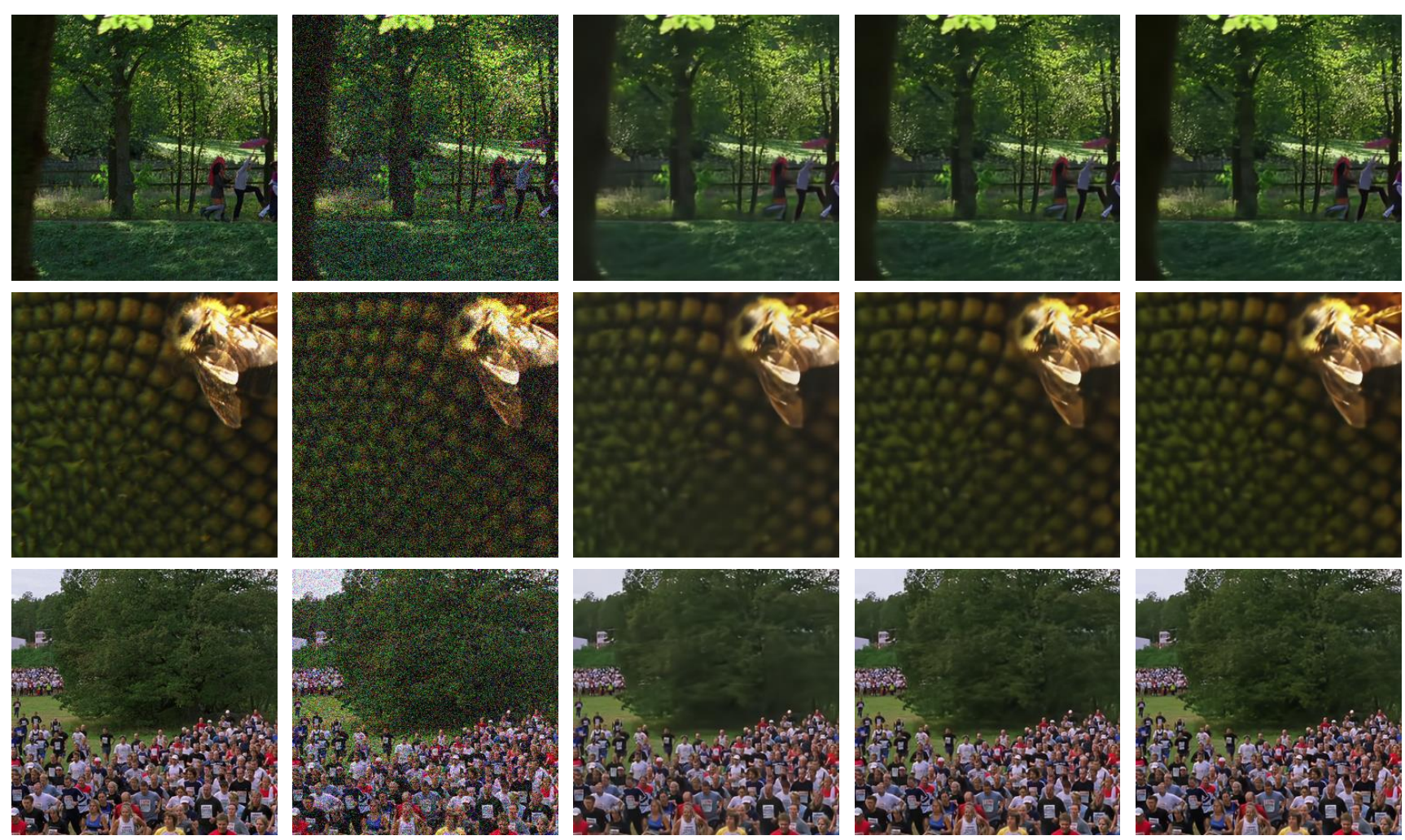

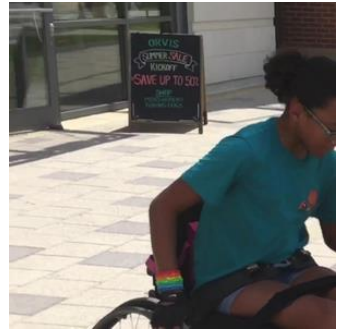

ground-truth

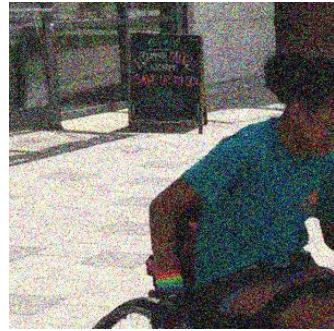

noisy

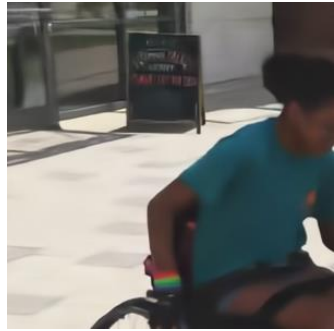

pre-trained

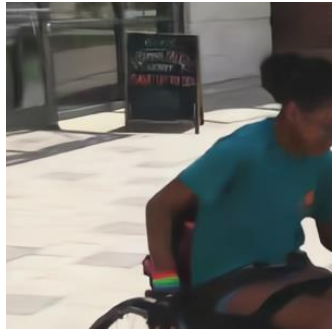

online

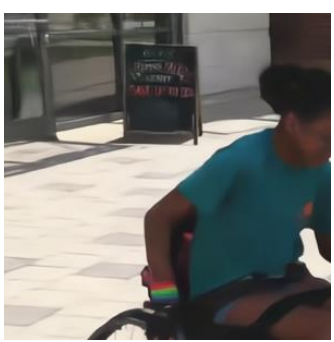

offline

Figure 2: Denoising results with FastDVDnet [25] on Derf testsets corrupted by Gaussian noise $(\sigma=40)$. Visual comparisons with our online and offline ( $K=10$ in Algorithm 1) update procedures.

son results. The input images are corrupted with high-level Gaussian noise $(\sigma=40)$, and FastDVDnet is fine-tuned by our offline and online restoration algorithms. We also provide real noise denoising results with fine-tuned RViDeNet in Fig. 3. Our methods can produce much better visual results and restore tiny details compared with the initially pretrained network.

Run-time. We report the run-time for a single update step on the NVIDIA Tesla V100 graphics unit. FastDVDnet and VNLnet take approximately 0.16 and 0.4 second to handle a $960 \times 540$ input frame with our online denoising algorithm in Algorithm 2; RViDeNet takes approximately 3.0 second to process the $256 \times 256$ input frame with our offline denoising method in Algorithm 1.

\subsection{Comparison with the frame-to-frame}

We compare our online denoising method with the frame-to-frame training algorithm [7], and their official code and parameters are used for the fine-tuning. As the baseline, a fully pre-trained DnCNN [28], which is trained with the Gaussian random noise $(\sigma=25)$ on the large external dataset is used, and then fine-tuned on the Derf dataset using frame-to-frame and our algorithms. For fair comparison, the same hyper-parameters (e.g., optimizer, learning rate, number of updates for each frame) are used to run the both frame-to-frame and our restoration algorithms; the comparison results are provided in Fig. 4.

First, we show that the frame-to-frame algorithm cannot outperform the baseline network when the noise distribution 


\begin{tabular}{|c|c|c|c|c|c|c|c|c|c|}
\hline Method & $\sigma$ & crowd & park joy & pedestrian & station & sunflower & touchdown & tractor & Average \\
\hline \multirow{9}{*}{ FastDVDnet } & \multirow{3}{*}{15} & $31.17 / 0.9208$ & $30.46 / 0.8959$ & $38.00 / 0.9522$ & $36.65 / 0.9323$ & $37.95 / 0.9525$ & $35.98 / 0.9109$ & $34.03 / 0.9263$ & $34.89 / 0.9273$ \\
\hline & & $31.32 / 0.9233$ & $30.72 / 0.9058$ & $38.20 / 0.9537$ & $36.83 / 0.9338$ & $38.38 / 0.9554$ & $36.06 / 0.9123$ & $34.26 / 0.9283$ & $35.11 / 0.9304$ \\
\hline & & $31.57 / 0.9263$ & $30.95 / 0.9108$ & $38.44 / 0.9549$ & $37.10 / 0.9363$ & $39.03 / 0.9596$ & $36.23 / 0.9138$ & $34.63 / 0.9320$ & $35.42 / 0.9334$ \\
\hline & \multirow{3}{*}{25} & $29.01 / 0.8813$ & $28.34 / 0.8373$ & $35.76 / 0.9328$ & $34.96 / 0.9050$ & $34.96 / 0.9306$ & $33.97 / 0.8667$ & $31.99 / 0.8946$ & $32.71 / 0.8926$ \\
\hline & & $29.32 / 0.8892$ & $28.79 / 0.8610$ & $36.27 / 0.9369$ & $35.12 / 0.9076$ & $36.14 / 0.9389$ & $34.09 / 0.8678$ & $32.32 / 0.8990$ & $33.15 / 0.9000$ \\
\hline & & $29.59 / 0.8943$ & $29.06 / 0.8719$ & $36.62 / 0.9393$ & $35.38 / 0.9107$ & $37.00 / 0.9455$ & $34.33 / 0.8703$ & $32.78 / 0.9051$ & $33.54 / 0.9053$ \\
\hline & \multirow{3}{*}{40} & $26.43 / 0.8159$ & $25.80 / 0.7441$ & $32.44 / 0.9003$ & $32.89 / 0.8634$ & $30.66 / 0.8884$ & $31.78 / 0.8022$ & $29.69 / 0.8490$ & $29.95 / 0.8376$ \\
\hline & & $27.21 / 0.8375$ & $26.82 / 0.7927$ & $33.93 / 0.9118$ & $33.34 / 0.8709$ & $33.39 / 0.9129$ & $32.18 / 0.8095$ & $30.32 / 0.8594$ & $31.03 / 0.8564$ \\
\hline & & $27.67 / 0.8509$ & $27.27 / 0.8192$ & $34.74 / 0.9190$ & $33.42 / 0.8717$ & $34.93 / 0.9263$ & $32.56 / 0.8140$ & $30.98 / 0.8697$ & $31.69 / 0.8679$ \\
\hline \multirow{9}{*}{ VNLnet } & \multirow{3}{*}{15} & $32.68 / 0.9373$ & $32.19 / 0.9200$ & $38.85 / 0.9567$ & $38.51 / 0.9501$ & $39.58 / 0.9628$ & $37.37 / 0.9347$ & $35.12 / 0.9378$ & $36.33 / 0.9428$ \\
\hline & & $32.83 / 0.9386$ & $32.47 / 0.9279$ & $38.94 / 0.9570$ & $38.60 / 0.9508$ & $39.87 / 0.9640$ & $37.41 / 0.9348$ & $35.30 / 0.9393$ & $36.49 / 0.9446$ \\
\hline & & $33.00 / 0.9401$ & $32.72 / 0.9328$ & $39.05 / 0.9576$ & $38.72 / 0.9515$ & $40.22 / 0.9661$ & $37.52 / 0.9350$ & $35.54 / 0.9416$ & $36.68 / 0.9464$ \\
\hline & \multirow{3}{*}{25} & $30.07 / 0.9013$ & $29.48 / 0.8651$ & $36.15 / 0.9353$ & $36.57 / 0.9263$ & $36.06 / 0.9430$ & $35.21 / 0.8976$ & $32.83 / 0.9072$ & $33.77 / 0.9108$ \\
\hline & & $30.34 / 0.9063$ & $30.00 / 0.8849$ & $36.58 / 0.9375$ & $36.69 / 0.9280$ & $37.37 / 0.9494$ & $35.32 / 0.8991$ & $33.11 / 0.9106$ & $34.20 / 0.9165$ \\
\hline & & $30.58 / 0.9105$ & $30.33 / 0.8970$ & $36.93 / 0.9397$ & $36.83 / 0.9293$ & $38.02 / 0.9539$ & $35.49 / 0.8994$ & $33.41 / 0.9142$ & $34.51 / 0.9206$ \\
\hline & \multirow{3}{*}{40} & $27.09 / 0.8366$ & $26.51 / 0.7724$ & $32.48 / 0.8992$ & $33.91 / 0.8817$ & $31.01 / 0.8975$ & $32.33 / 0.8122$ & $30.09 / 0.8569$ & $30.49 / 0.8509$ \\
\hline & & $27.85 / 0.8536$ & $27.61 / 0.8153$ & $33.78 / 0.9078$ & $34.36 / 0.8879$ & $34.09 / 0.9231$ & $32.71 / 0.8199$ & $30.71 / 0.8672$ & $31.57 / 0.8678$ \\
\hline & & $28.35 / 0.8648$ & $28.18 / 0.8425$ & $34.71 / 0.9154$ & $34.64 / 0.8910$ & $35.62 / 0.9343$ & $33.06 / 0.8220$ & $31.33 / 0.8760$ & $32.27 / 0.8780$ \\
\hline
\end{tabular}

Table 1: Denoising results with FastDVDnet [25] and VNLnet [6] on the Derf testset with different Gaussian noise levels $(\sigma=15,25,40)$. For each network architecture and each noise level, the PSNR and SSIM results of the baseline, online learning (Algorithm 2) and offline learning (Algorithm 1) are listed in each box from top to bottom. The best average results are written in bold letters.

\begin{tabular}{|c|c|c|c|c|c|c|c|c|c|}
\hline Method & $\sigma$ & chamaleon & giant-slalom & girl-dog & hoverboard & monkeys-trees & salsa & subway & Average \\
\hline \multirow{9}{*}{ FastDVDnet } & \multirow{3}{*}{15} & $36.65 / 0.9697$ & "40.79/0.9685 & 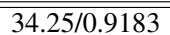 & $39.55 / 0.9613$ & ב31.59/0.9567 & (33.41/0.9440 & 37.56/0.9246 & $36.26 / 0.9490$ \\
\hline & & $36.73 / 0.9702$ & $41.08 / 0.9708$ & $34.29 / 0.9202$ & $39.63 / 0.9617$ & $31.63 / 0.9569$ & $33.62 / 0.9519$ & $39.20 / 0.9601$ & $36.60 / 0.9560$ \\
\hline & & $36.92 / 0.9711$ & $41.29 / 0.9717$ & $34.35 / 0.9214$ & $39.71 / 0.9616$ & $31.75 / 0.9578$ & $33.84 / 0.9626$ & $40.03 / 0.9741$ & $36.84 / 0.9601$ \\
\hline & \multirow{3}{*}{25} & $33.98 / 0.9534$ & $38.67 / 0.9550$ & $31.61 / 0.8590$ & $37.36 / 0.9486$ & $28.71 / 0.9161$ & $30.17 / 0.9002$ & $33.48 / 0.8737$ & $33.43 / 0.9151$ \\
\hline & & $34.22 / 0.9550$ & $38.94 / 0.9577$ & $31.73 / 0.8639$ & $37.54 / 0.9494$ & $28.74 / 0.9164$ & $30.59 / 0.9143$ & $36.32 / 0.9334$ & $34.01 / 0.9272$ \\
\hline & & $34.53 / 0.9567$ & $39.16 / 0.9588$ & $31.88 / 0.8679$ & $37.82 / 0.9504$ & $28.84 / 0.9184$ & $30.93 / 0.9328$ & $37.95 / 0.9637$ & $34.44 / 0.9355$ \\
\hline & \multirow{3}{*}{40} & $30.97 / 0.9263$ & $36.53 / 0.9384$ & $29.01 / 0.7785$ & $34.82 / 0.9315$ & $26.29 / 0.8506$ & $26.76 / 0.8162$ & $28.83 / 0.8070$ & $30.46 / 0.8640$ \\
\hline & & $31.58 / 0.9311$ & $36.84 / 0.9410$ & $29.44 / 0.7907$ & $35.32 / 0.9338$ & $26.32 / 0.8529$ & $27.65 / 0.8500$ & $32.88 / 0.8885$ & $31.43 / 0.8840$ \\
\hline & & $32.30 / 0.9364$ & $37.16 / 0.9428$ & $29.84 / 0.7985$ & $35.92 / 0.9361$ & $26.38 / 0.8546$ & $28.26 / 0.8819$ & $35.85 / 0.9505$ & $32.25 / 0.9001$ \\
\hline \multirow{9}{*}{ VNLnet } & \multirow{3}{*}{15} & $37.30 / 0.9724$ & $42.31 / 0.9751$ & $35.68 / 0.9407$ & $39.83 / 0.9626$ & $34.87 / 0.9792$ & $34.04 / 0.9461$ & $37.60 / 0.9188$ & $37.37 / 0.9564$ \\
\hline & & $37.37 / 0.9725$ & $42.32 / 0.9751$ & $35.73 / 0.9418$ & $39.82 / 0.9621$ & $34.94 / 0.9794$ & $34.21 / 0.9517$ & $39.42 / 0.9522$ & $37.69 / 0.9621$ \\
\hline & & $37.50 / 0.9734$ & $42.40 / 0.9753$ & $35.81 / 0.9428$ & $39.88 / 0.9621$ & $35.02 / 0.9798$ & $34.39 / 0.9616$ & $40.41 / 0.9720$ & $37.97 / 0.9667$ \\
\hline & \multirow{3}{*}{25} & $34.46 / 0.9567$ & $39.75 / 0.9604$ & $32.76 / 0.8887$ & $37.58 / 0.9501$ & $31.96 / 0.9591$ & $30.60 / 0.9015$ & $32.88 / 0.8642$ & $34.28 / 0.9258$ \\
\hline & & $34.66 / 0.9576$ & $39.81 / 0.9606$ & $32.93 / 0.8947$ & $37.66 / 0.9497$ & $32.02 / 0.9596$ & $31.03 / 0.9134$ & $35.66 / 0.9085$ & $34.83 / 0.9349$ \\
\hline & & $34.98 / 0.9595$ & $39.91 / 0.9610$ & $33.09 / 0.8973$ & $37.89 / 0.9500$ & $32.09 / 0.9598$ & $31.40 / 0.9314$ & $37.98 / 0.9598$ & $35.33 / 0.9456$ \\
\hline & \multirow{3}{*}{40} & $31.26 / 0.9302$ & $37.23 / 0.9425$ & $29.71 / 0.8061$ & $34.96 / 0.9335$ & $29.13 / 0.9208$ & $27.05 / 0.8198$ & $28.55 / 0.8052$ & $31.13 / 0.8797$ \\
\hline & & $31.81 / 0.9338$ & $37.41 / 0.9431$ & $30.24 / 0.8224$ & $35.31 / 0.9343$ & $29.23 / 0.9236$ & $27.99 / 0.8510$ & $32.05 / 0.8639$ & $32.01 / 0.8960$ \\
\hline & & $32.57 / 0.9391$ & $37.65 / 0.9438$ & $30.71 / 0.8306$ & $35.93 / 0.9366$ & $29.30 / 0.9242$ & $28.69 / 0.8798$ & $35.65 / 0.9433$ & $32.93 / 0.9139$ \\
\hline
\end{tabular}

Table 2: Denoising results with FastDVDnet [25] and VNLnet [6] on the DAVIS testset with different Gaussian noise levels $(\sigma=15,25,40)$. For each network architecture and each noise level, the PSNR and SSIM results of the baseline, online learning (Algorithm 2) and offline learning (Algorithm 1) are listed in each box from top to bottom. The best average results are written in bold letters.

of the test video is identical to that in the large external train set (i.e., $\sigma=25$ ). By contrast, our online restoration algorithm can still elevate the denoising performance compared with the fully pre-trained baseline by adapting the network parameter to the specific input video (Fig. 4 (a)). Next, compared with the baseline, the frame-to-frame algorithm and our approach can improve denoising quality by a large margin when the noise distribution of the input video (i.e., $\sigma=$ 40 ) is different from that of the training dataset (Fig. 4 (b)).
However, when the input video includes large motion displacement, the frame-to-frame algorithm fails in estimating accurate optical flow and thus can show worse performance than the baseline; by contrast, our algorithm predicts consistently better results because ours does not rely on the optical flow (solid blue lines in Fig. 4 (c)).

In the comparison with the frame-to-frame method, our algorithm uses additional information regarding the noise distribution of the test input but shows considerably bet- 


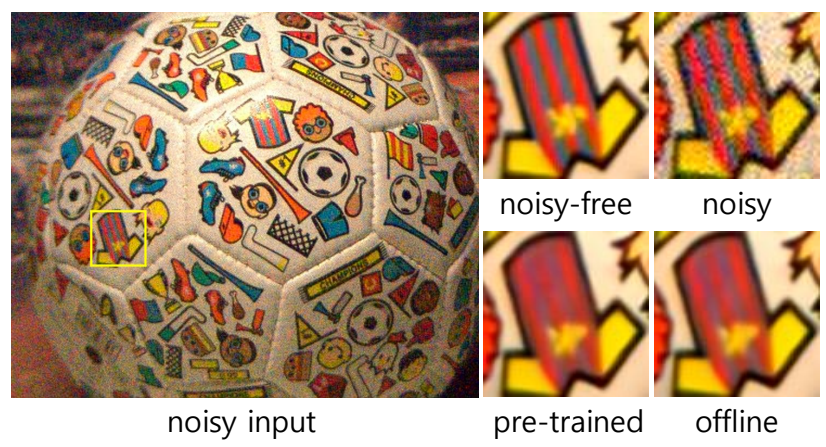

Figure 3: Denoising results with RViDeNet on the CRVD [27] dataset which includes real noise. We use $K$ $=10$, for our offline denoising algorithm.

\begin{tabular}{|c|c|c|c|}
\hline & Noisy & RViDeNet & Ours \\
\hline PSNR & 31.79 & 39.95 & $\mathbf{4 0 . 1 3}$ \\
\hline SSIM & 0.7517 & 0.9792 & $\mathbf{0 . 9 7 9 5}$ \\
\hline
\end{tabular}

Table 3: Denoising results with RViDeNet on the CRVD [27] dataset with real noise. The proposed offline learning method (Algorithm 1) show the quantitatively better results than the baseline (RViDeNet) and the results are written in bold letters.

ter denoising results. By contrast, the frame-to-frame algorithm requires additional resources and longer run-time to compute optical flow among video frames.

\section{Conclusion}

In this work, we present a new training algorithm for video denoising; this algorithm is straightforward and easy to train and produces state-of-the-art denoising results. Our training approach is based on the self-supervision and thus allows the network to adapt its pre-trained parameter for the given specific input video without using ground-truth clean frames. As we use the restored version of the input noisy frames rendered by the pre-trained denoiser as our fine-tuning target (pseudo clean images), we call the proposed algorithm "restore-from-restored." Moreover, in contrast to conventional video restoration approaches, we restore the clean images without using accurate optical flow. We describe how the proposed training algorithm can exploit recurring patches among input video frames and improve the denoising performance. We also demonstrate the superiority of the proposed algorithm and show considerable improvements on the various benchmark datasets.

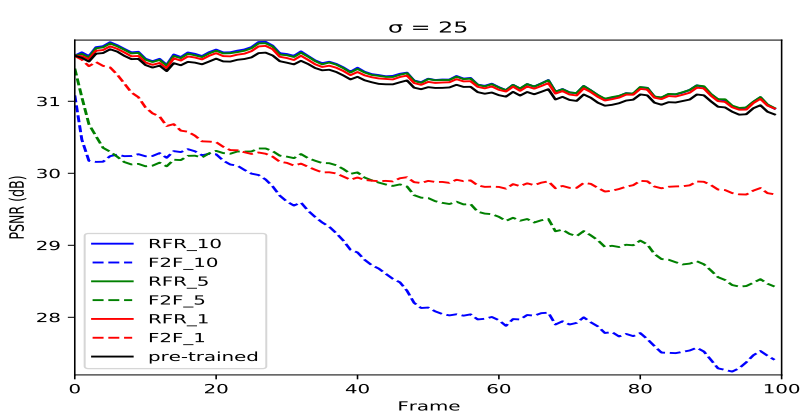

(a)

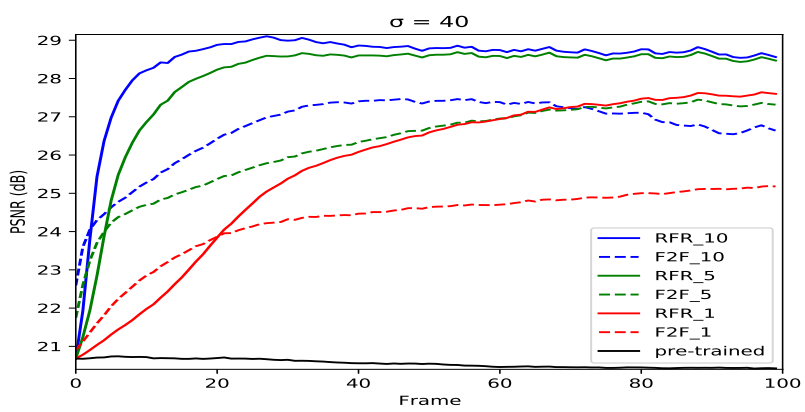

(b)

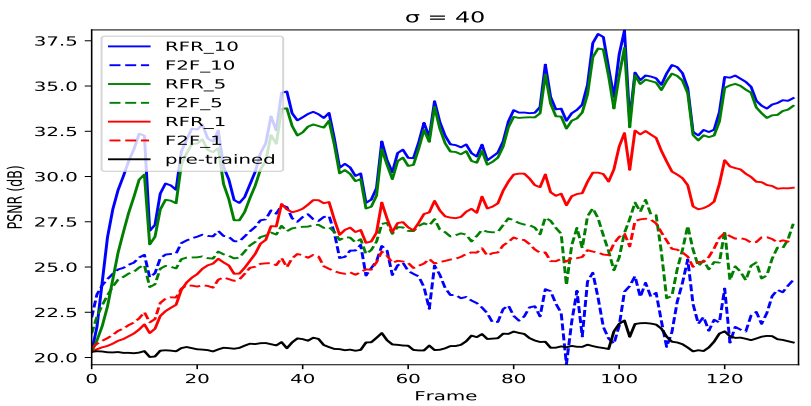

(c)

Figure 4: Comparisons of restore-from-restored (RFR) and frame-to-frame (F2F) online fine-tuning methods. The number $i$ in "F2F_ $i$ " or "RFR $i$ " indicates the number of updates for each frame [7]. (a) Denoising results when the test input noise level is higher $(\sigma=40)$ than that in training $(\sigma=25)$. (b) Denoising results when noise level during the test and training stages is identical. (c) Denoising results on a video with large motions when noise level during the test and training stages is identical.

\section{References}

[1] Saeed Anwar and Nick Barnes. Real image denoising with feature attention. In Proceedings of the IEEE International Conference on Computer Vision (ICCV), 2019. 2

[2] Joshua Batson and Loic Royer. Noise2self: Blind denoising by self-supervision. In International Conference on Machine Learning (ICML), 2019. 2, 3

[3] Jose Caballero, Christian Ledig, Andrew Aitken, Alejan- 
dro Acosta, Johannes Totz, Zehan Wang, and Wenzhe Shi. Real-time video super-resolution with spatio-temporal networks and motion compensation. In Proceedings of the IEEE Conference on Computer Vision and Pattern Recognition $(C V P R), 2017.3$

[4] Taco Cohen and Max Welling. Group equivariant convolutional networks. In International Conference on Machine Learning (ICML), 2016. 4

[5] Jifeng Dai, Haozhi Qi, Yuwen Xiong, Yi Li, Guodong Zhang, Han Hu, and Yichen Wei. Deformable convolutional networks. In Proceedings of the IEEE International Conference on Computer Vision (ICCV), 2017. 2

[6] Axel Davy, Thibaud Ehret, Jean-Michel Morel, Pablo Arias, and Gabriele Facciolo. Non-local video denoising by $\mathrm{cnn}$. In IEEE International Conference on Image Processing (ICIP), 2019. 2, 3, 5, 7

[7] Thibaud Ehret, Axel Davy, Jean-Michel Morel, Gabriele Facciolo, and Pablo Arias. Model-blind video denoising via frame-to-frame training. In Proceedings of the IEEE Conference on Computer Vision and Pattern Recognition (CVPR), 2019. 1, 2, 3, 4, 6, 8

[8] Ruohan Gao and Kristen Grauman. On-demand learning for deep image restoration. In Proceedings of the IEEE International Conference on Computer Vision (ICCV), 2017. 2

[9] Daniel Glasner, Shai Bagon, and Michal Irani. Superresolution from a single image. In Proceedings of the IEEE International Conference on Computer Vision (ICCV), 2009. 1

[10] Shi Guo, Zifei Yan, Kai Zhang, Wangmeng Zuo, and Lei Zhang. Toward convolutional blind denoising of real photographs. In Proceedings of the IEEE Conference on Computer Vision and Pattern Recognition (CVPR), 2018. 2

[11] Jia-Bin Huang, Abhishek Singh, and Narendra Ahuja. Single image super-resolution from transformed self-exemplars. In Proceedings of the IEEE Conference on Computer Vision and Pattern Recognition (CVPR), 2015. 1

[12] Jun-Jie Huang, Tianrui Liu, Pier Luigi Dragotti, and Tania Stathaki. Srhrf+: Self-example enhanced single image superresolution using hierarchical random forests. In Proceedings of the IEEE Conference on Computer Vision and Pattern Recognition Workshops, pages 71-79, 2017. 1

[13] Tae Hyun Kim, Mehdi SM Sajjadi, Michael Hirsch, and Bernhard Schölkopf. Spatio-temporal transformer network for video restoration. In Proceedings of the European Conference on Computer Vision (ECCV), 2018. 3

[14] Alexander Krull, Tim-Oliver Buchholz, and Florian Jug. Noise2void-learning denoising from single noisy images. In Proceedings of the IEEE Conference on Computer Vision and Pattern Recognition (CVPR), 2019. 2

[15] Stamatios Lefkimmiatis. Non-local color image denoising with convolutional neural networks. In Proceedings of the IEEE Conference on Computer Vision and Pattern Recognition (CVPR), pages 5882-5891, 2016. 2

[16] Jaakko Lehtinen, Jacob Munkberg, Jon Hasselgren, Samuli Laine, Tero Karras, Miika Aittala, and Timo Aila. Noise2Noise: Learning image restoration without clean data. In International Conference on Machine Learning (ICML), volume $80,2018.1,2,3,4$
[17] Ding Liu, Bihan Wen, Yuchen Fan, Chen Change Loy, and Thomas S. Huang. Non-local recurrent network for image restoration. In Advances in Neural Information Processing Systems (NIPS), pages 1680-1689, 2018. 2, 3

[18] Matteo Maggioni, Giacomo Boracchi, Alessandro Foi, and Karen Egiazarian. Video denoising, deblocking, and enhancement through separable 4-d nonlocal spatiotemporal transforms. IEEE Transactions on Image Processing, 21(9):3952-3966, 2012. 1

[19] Mona Mahmoudi and Guillermo Sapiro. Fast image and video denoising via nonlocal means of similar neighborhoods. IEEE signal processing letters, 12(12):839-842, 2005. 1

[20] Jordi Pont-Tuset, Federico Perazzi, Sergi Caelles, Pablo Arbeláez, Alex Sorkine-Hornung, and Luc Van Gool. The 2017 davis challenge on video object segmentation. arXiv:1704.00675, 2017. 5

[21] Mehdi SM Sajjadi, Raviteja Vemulapalli, and Matthew Brown. Frame-recurrent video super-resolution. In Proceedings of the IEEE Conference on Computer Vision and Pattern Recognition, pages 6626-6634, 2018. 3

[22] Tamar Rott Shaham, Tali Dekel, and Tomer Michaeli. Singan: Learning a generative model from a single natural image. In Proceedings of the IEEE International Conference on Computer Vision (ICCV), 2019. 3

[23] Oded Shahar, Alon Faktor, and Michal Irani. Space-time super-resolution from a single video. In Proceedings of the IEEE Conference on Computer Vision and Pattern Recognition (CVPR), 2011. 3

[24] Assaf Shocher, Nadav Cohen, and Michal Irani. "zero-shot" super-resolution using deep internal learning. In Proceedings of the IEEE Conference on Computer Vision and Pattern Recognition (CVPR), 2018. 3

[25] Matias Tassano, Julie Delon, and Thomas Veit. Fastdvdnet: Towards real-time deep video denoising without flow estimation. In Proceedings of the IEEE Conference on Computer Vision and Pattern Recognition (CVPR), 2020. 2, 5, 6, 7

[26] Junyuan Xie, Linli Xu, and Enhong Chen. Image denoising and inpainting with deep neural networks. In Advances in Neural Information Processing Systems (NIPS), pages 341349, 2012. 2

[27] Huanjing Yue, Cong Cao, Lei Liao, Ronghe Chu, and Jingyu Yang. Supervised raw video denoising with a benchmark dataset on dynamic scenes. In Proceedings of the IEEE Conference on Computer Vision and Pattern Recognition (CVPR), 2020. 2, 5, 8

[28] Kai Zhang, Wangmeng Zuo, Yunjin Chen, Deyu Meng, and Lei Zhang. Beyond a gaussian denoiser: Residual learning of deep cnn for image denoising. IEEE Transactions on Image Processing, 26:3142-3155, 2017. 2, 6

[29] Kai Zhang, Wangmeng Zuo, Shuhang Gu, and Lei Zhang. Learning deep cnn denoiser prior for image restoration. In Proceedings of the IEEE Conference on Computer Vision and Pattern Recognition (CVPR), pages 2808-2817, 2017. 2

[30] Kai Zhang, Wangmeng Zuo, and Lei Zhang. Ffdnet: Toward a fast and flexible solution for cnn based image de- 
noising. IEEE Transactions on Image Processing, 27:46084622, 2018. 2

[31] Yulun Zhang, Kunpeng Li, Kai Li, Bineng Zhong, and Yun $\mathrm{Fu}$. Residual non-local attention networks for image restoration. In Proceedings of the International Conference on Learning Representations (ICLR), 2019. 2, 3

[32] Yulun Zhang, Yapeng Tian, Yu Kong, Bineng Zhong, and Yun Fu. Residual dense network for image restoration. IEEE Transactions on Pattern Analysis and Machine Intelligence (PAMI), 2020. 2 